\title{
ATRIBUTOS DE DIFERENCIACIÓN Y VALORACIÓN EN VAINILLA MEXICANA, REGIÓN DE ORIGEN
}

\author{
Ariadna Barrera $^{a^{*}}$ Anastacio Espejel ${ }^{a}$ y María Guadalupe Pérez ${ }^{a}$ \\ ${ }^{a}$ Universidad Autónoma Chapingo (Estado de México, ariadna.barrera@gmail.com)
}

\section{RESUMEN}

La vainilla es uno de los productos aromáticos más emblemáticos debido al proceso de producción y beneficiado tradicional que respalda la denominación de origen. En los últimos años ha incrementado el valor de la vainilla, sin embargo, a nivel nacional el consumo es bajo, debido al elevado y fluctuante precio y a la escasa valorización de los consumidores. El objetivo del trabajo fue identificar atributos de valoración y diferenciación en una muestra de consumidores. Se diseñó una encuesta y se aplicó a 100 consumidores locales y no locales. Se utilizó la metodología de significados de compra para identificar los significados que los consumidores nacionales le otorgan a la vainilla; para la valoración económica se utilizó el método de valoración contingente. Los resultados indican que los significados de compra están relacionados con el aroma (27\%), alimento (20\%), los procesos (15\%) principalmente. Los consumidores valoran que la vainilla mexicana sea nacional, natural, producida por pequeños productores y que tenga denominación de origen. La mayor disposición a pagar fue por vainilla con denominación de origen. Se concluye que existe un grupo de consumidores que valoran y están dispuestos a pagar un sobreprecio.

Palabras clave: alimento tradicional, vainilla mexicana, valorización, atributos sensoriales.

\section{INTRODUCCIÓN}

La vainilla (Vanilla planifolia Jacks Ex. Adrews) es una de las especias más comercializadas en el mercado internacional debido a su aroma insustituible. Es una orquídea cuyo fruto verde es sometido a un proceso de beneficiado tradicional, que consiste en un proceso artesanal compuesto por cuatro etapas: asoleado, sudado, secado y acondicionado. El proceso es realizado por un maestro beneficiador quien tiene muchos años de experiencia y preserva los conocimientos transmitidos por generaciones.

La vainilla mexicana obtuvo la denominación de origen en 2011 sustentada en la Norma Oficial NOM-182SCFI-2011, "Vainilla de Papantla. Extractos y derivados. Especificaciones, información comercial y métodos de ensayo (prueba)". La vainilla mexicana se ha posicionado como la mejor en el ámbito mundial por su perfil sensorial, es demandada por la industria alimentaria. Es un recurso fitogenético asociado ancestralmente a las culturas originarias, se tienen registros de su uso alimentario y medicinal (Deanne, 2005). Si bien en México el consumo per cápita de vainilla es bajo, en los últimos años se ha registrado un aumento en el consumo por la industria de alimentos.

Los alimentos tradicionales son productos con un arraigo cultural e identitario y cuyos atributos tangibles e intangibles están asociado con el territorio (Guerrero et al. ,2009). Los atributos sensoriales de los productos tradicionales están relacionados con los procesos, los implementos, las prácticas, los conocimientos ancestrales que conlleva su elaboración. Por tanto, los productos tradicionales son una expresión de la cultura, historia y el modo de vida de una región. Son considerados saludables y con gran aportación nutricional, y por otro lado contribuyen a conservar elementos de la herencia cultural (Trichopoulou et al., 2007).

Los conocimientos entorno a su domesticación, conservación, procesamiento y uso de la vainilla, están estrechamente asociados a las culturas y a los pueblos originarios asentados en las regiones productoras (Del Ángel Pérez y Mendoza, 2002). Los atributos sensoriales de la vainilla están atribuidos a las condiciones físico-climáticas de la región, a prácticas agrícolas tradicionales en la producción y el proceso de beneficiado de la vainilla, así como los utensilios y materiales empleados. Podemos afirmar que la vainilla mexicana es un alimento tradicional con gran riqueza cultural. Por lo tanto, el objetivo del presente trabajo fue identificar atributos de valoración y diferenciación de la vainilla mexicana en una muestra de consumidores.

De acuerdo con Jauregui et al. (2021) los alimentos tradicionales son caracterizados por un conjunto de atributos que pueden estar asociados a una evaluación subjetiva, a partir de la cual es posible aproximar una función de utilidad y determinar elementos para su valoración. La valorización de los alimentos tradicionales parte de comprender cómo los consumidores perciben los productos, cómo influyen sus necesidades y cómo toman decisiones basadas en esas necesidades, así como la disposición a pagar por un producto con características típicas y únicas diferenciadoras (Van Kleef et al., 2005). Existen factores que intervienen en el consumo de alimentos como el origen del producto, el proceso de fabricación, vinculación a territorios, funcionalidad y valores éticos (Vanhonacker et al., 2013). 
La disposición a pagar de un consumidor está influenciada por sus gustos y preferencias individuales, sus ingresos, actitudes y percepciones de los diferentes tipos de productos, así como las características demográficas y del hogar (Lanfranchi et al., 2019). Existen estudios sobre la disposición a pagar por productos tradicionales mexicanos (Jauregui et al., 2021; Barrera et al., 2019; Jaramillo et al., 2018) los cuales contribuyen a la revalorización y segmentación del mercado para este grupo de alimentos emblemáticos vinculados al territorio.

\section{METODOLOGÍA}

Para el análisis sensorial se empleó el Perfil Flash (Delarue y Sieffermann, 2003); se integró un panel de nueve productores de vainilla. Se seleccionaron cuatro tipos de vainilla beneficiada provenientes de distintas comunidades pertenecientes a la región del Totonacapan en los estados de Veracruz y Puebla. Las variables que fueron evaluadas por el panel de expertos fueron: color café (Homogeneidad), brillantez, flexibilidad, aroma a vainilla, aroma fermentado, aroma a madera, aroma a dulce, aroma a chocolate, aroma a fruta, aroma floral, aroma a tabaco y aroma a anís.

En la primera etapa del perfil Flash, se les proporcionó a los panelistas una vaina de vainilla con la finalidad de generar de manera individual una lista de atributos que les permitieran describirla; en una segunda etapa, se obtuvo por consenso una lista de los atributos de la vainilla. En la tercera etapa, los panelistas jerarquizaron cada uno de los atributos para las cuatro muestras de vainilla, empleando una escala ordinal de cuatro puntos (1, menos intenso y 4, más intenso). Los atributos generados por cada panelista se analizaron con un diseño completo al azar, con la finalidad de seleccionar los atributos que mostraran diferencia significativa $(\mathrm{p} \leq 0.05)$ entre tratamientos. Se empleó el análisis de componentes principales para establecer la asociación entre atributos evaluados y los cuatro tipos de vainilla.

Se diseñó una encuesta y se aplicó a una muestra de 100 consumidores locales y no locales. El estudio conceptual se realizó mediante las palabras asociadas con la vainilla que fueron referenciadas por los consumidores de dos zonas de consumo (Veracruz y zona centro) ante la pregunta ¿cuáles son las palabras que evoca con la palabra vainilla?; a partir de ellas se crearon nueve categorías: aroma, ruralidad, tradicionalidad, alimentos, economía, identidad, procesos, atributo de sabor, bebidas. Las categorías se analizaron mediante una comparación de K proporciones para los consumidores de cada zona de consumo.

La estimación de la disposición a pagar (DAP) se realizó mediante el estudio de valoración contingente, que permite conocer la DAP del consumidor por cambios o conservación de ciertos atributos de los alimentos en donde existe un juicio de valoración (Rousseau y Vranken, 2013). La pregunta formulada fue ¿está dispuesto a pagar un sobreprecio (porcentaje) por vainilla con las siguientes características: origen mexicano, vainilla natural, vainilla mexicana con denominación de origen, beneficiada por pequeños productores, orgánica.

\section{RESULTADOS}

El análisis de componentes principales describe los atributos que presenta la vainilla de Papantla los cuales son: Aroma Tabaco, Anís, Madera, Aroma a Floral y Brillantez, el atributo que más la caracteriza es el aroma a tabaco. Los atributos que caracteriza la vainilla de San José Acateno indican un mayor aroma a: Dulce, Chocolate y Vainilla, también describieron a la vainilla con un aroma fermentado, atributos organolépticos como flexibilidad y color café. Por otro lado, la vainilla de Coyuxquihui y Primero de mayo no fueron asociadas con ningún atributo evaluado (Gráfico 1). 
Biplot (ejes F1 y F2: $89.01 \%$ )

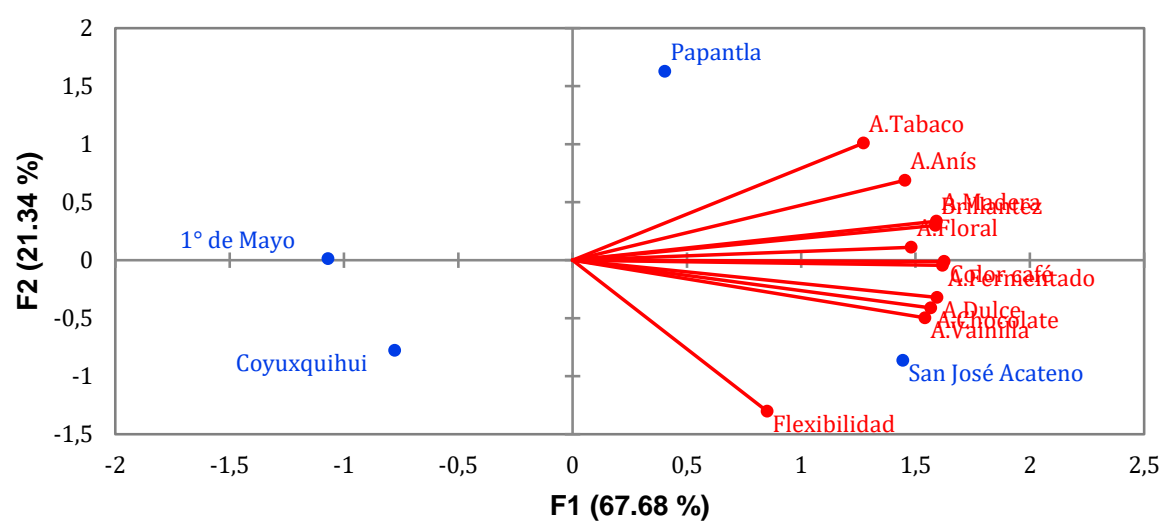

Fuente: Elaboración propia

Gráfico 1. Atributos consensuados por panelistas en el perfil flash

Las categorías para la zona Veracruz que registraron significancia fueron: aroma y alimentos. Para la zona centro, las categorías que registraron significancia fueron: Aroma, Alimentos y Ruralidad. En la categoría de Tradicionalidad los consumidores de la zona Centro no asociaron palabras a pesar de que en el cultivo de vainilla tiene una gran importancia cultural, sin embargo, el conocimiento de vainilla como recurso fitogenético se reconoce más en la zona productora.

Cuadro 1. Significancia por categoría en cada grupo de consumidores

\begin{tabular}{lll}
\hline Categoría & Veracruz & Centro \\
\hline Aroma & $\mathbf{2 7 . 3 2} \mathbf{c}$ & $\mathbf{1 9 . 6 0} \mathbf{a}$ \\
Ruralidad & $6.01 \mathrm{a}$ & 15.68 \\
Tradicionalidad & $9.28 \mathrm{ab}$ & 0.00 \\
Alimentos & $\mathbf{2 0 . 7 6} \mathbf{~ b c}$ & $\mathbf{2 1 . 5 6} \mathbf{a}$ \\
Economía & $8.19 \mathrm{ab}$ & $11.76 \mathrm{a}$ \\
Identidad & $4.37 \mathrm{a}$ & $5.88 \mathrm{a}$ \\
Procesos & $5.46 \mathrm{a}$ & $\mathbf{1 5 . 6 8} \mathbf{a}$ \\
Atributos de sabor & $8.74 \mathrm{ab}$ & $5.88 \mathrm{a}$ \\
Bebidas & $9.83 \mathrm{~b}$ & $3.92 \mathrm{a}$ \\
\hline
\end{tabular}

Fuente: Elaboración Propia

${ }^{\mathrm{Z}}$ Proporciones con la misma letra dentro de columnas, son estadísticamente iguales $(\mathrm{p} \leq 0.05)$

Las características socio-demográficas género y escolaridad fueron significativas en la disposición a pagar por vainilla con denominación de origen. Los resultados indican que el género masculino y los consumidores con mayor escolaridad están dispuesto a pagar un sobreprecio por una vainilla que cuente con denominación y sea producidos por pequeños productores.

En relación con la disposición a pagar el $72 \%$ de los consumidores afirmaron estar dispuestos a pagar entre 5 y $10 \%$ más por la vainilla con denominación de origen (Cuadro 3).

Cuadro 3. Porcentajes de consumidores dispuestos a pagar un sobreprecio por tipo de vainilla

\begin{tabular}{llllll}
\hline \multicolumn{1}{c}{ Atributo } & $0 \%$ & $5 \%$ & $10 \%$ & $20 \%$ & $30 \%$ \\
\hline Vainilla de Origen Mexicano & $7.87 \mathrm{a}$ & $34.83 \mathrm{~b}$ & $34.83 \mathrm{a}$ & $14.61 \mathrm{a}$ & $7.87 \mathrm{a}$ \\
Vainilla Natural & $5.62 \mathrm{a}$ & $30.34 \mathrm{ab}$ & $40.45 \mathrm{a}$ & $15.73 \mathrm{a}$ & $7.87 \mathrm{a}$ \\
$\begin{array}{l}\text { Vainilla mexicana con denominación de } \\
\text { Origen }\end{array}$ & $\mathbf{4 . 4 9} \mathrm{a}$ & $\mathbf{3 7 . 0 8 b}$ & $\mathbf{3 4 . 8 3 a}$ & $\mathbf{1 5 . 7 3 a}$ & $\mathbf{7 . 8 7} \mathbf{a}$ \\
$\begin{array}{l}\text { Vainilla producida beneficiada por } \\
\text { pequeños productores }\end{array}$ & $3.6 \mathrm{a}$ & $22.47 \mathrm{ab}$ & $43.82 \mathrm{a}$ & $17.98 \mathrm{a}$ & $11.24 \mathrm{a}$ \\
Vainilla Orgánica & $6.0 \mathrm{ab}$ & $13.48 \mathrm{a}$ & $23.60 \mathrm{a}$ & $20.22 \mathrm{a}$ & $11.24 \mathrm{a}$ \\
\hline
\end{tabular}

Fuente: Elaboración propia

${ }^{\mathrm{Z}}$ Proporciones con la misma letra dentro de columnas, son estadísticamente iguales $(\mathrm{p} \leq 0.05)$ 


\section{CONCLUSIÓN}

Las características sociodemográficas de los consumidores tales como el género y el nivel de escolaridad influyen significativamente en la disposición a pagar un precio mayor por vainilla. La vainilla mexicana con Denominación de Origen presentó una mayor disposición a pagar un sobreprecio entre $5 \%-10 \%$. Los consumidores de la región productora asociaron la vainilla con el aroma y los alimentos, mientras que los consumidores de la zona centro destacan además la categoría de procesos.

\section{BIBLIOGRAFÍA}

Barrera, R.A., Cuevas, R.V. y Espejel, G.A. (2019). Factores de valoración en consumidores de mezcal en Oaxaca. Revista de Estudios Sociales. 29 (54): 1-22. https://dx.doi.org/10.24836/es.v29i54.811

Deanne, B. J, MS, BS, BA. (2005). Historical perspective: Vanilla as a Medicinal Plant. ELSEVIER. 129131. doi:10.1016/j.sigm.2006.03.001

Del Ángel, P.A.L., y Mendoza B.M.A. (2002). Familia totonaca, expresión cultural y sobrevivencia. Papeles de población, 8(32), 95-120.

Guerrero, L., Guàrdia, M. D., Xicola, J., Verbeke, W., Vanhonacker, F., Zakowska-Biemans, S. \& Scalvedi, M. L. (2009). Consumer-driven definition of traditional food products and innovation in traditional foods. A qualitative cross-cultural study. Appetite, 52(2), 345-354.

Jaramillo, V. J.L., Vargas, L.S. y Rojas, J. A. (2018). Valoración y contingente y disponibilidad a pagar por atributos intangible en carne de bovino. Revista Mexicana de Ciencias Pecuarias. 9 (1): 14-31. http://dx.doi.org/

Jauregui, G. C. Z., Espejel, G. A. y Hernández, M. A. (2021). Valoración y disposición a pagar por los consumidores de elote en México. Rev. Fac. Agron. (LUZ). 38: 441-461. Abril-Junio. DOI: https://doi.org/10.47280/RevFacAgron(LUZ).v38.n2.12

Lanfranchi, M., E. Schimmenti, M.G. Campolo and C. Giannetto. (2019). The willingness to pay of Sicilian consumer for a wine obtained with sustainable production method: an estimate through an ordered probit sample-selection model. Wine Economics and Policy. 8(2):203-215.

Rousseau, S., and L. Vranken. (2013). Green market expansion by reducing information asymmetries: Evidence for labeled organic food products. Food Policy. 40:31-43.

Trichopoulou, A., Soukara, S. y Vasilopoulou, E. (2007). Traditional food: Science and society perspective. Trends in Food Science and Technology, (18): 420-427.

Van Kleef, E., H.C.M., Van, Trijp and Luning, P. (2005). Consumer research in the early stages of new product development: A critical review of methods and techniques. Food Qual. Prefer. 16:181-201. 